Journal of Applied AnALysis

Vol. 6, No. 2 (2000), pp. 295-302

\title{
ON CONTINUITY OF MEASURABLE COCYCLES
}

\author{
G. GUZIK \\ Received January 18, 2000 and, in revised form, July 27, 2000
}

\begin{abstract}
It is proved that every measurable, non-vanishing cocycle defined on the product of $(0, \infty)$ and an arbitrary compact metric space is continuous. Some other sufficient conditions for continuity of a cocycle are also given.
\end{abstract}

Consider functions $F$ satisfying the translation equation

$$
F(s+t, x)=F(t, F(s, x))
$$

and real or complex valued solutions of the equation

$$
G(s+t, x)=G(s, x) G(t, F(s, x)) .
$$

The two functions $F$ and $G$ satisfying $(\mathrm{T})$ and $(\mathrm{G})$, respectively, are known as an abstract automaton (see [11]). The equation $(\mathrm{G}$ ) occurs also (in the additive form) in ergodic theory for changing velocity in flows (see [15]). It plays a fundamental role in solving the problem of embeddability of linear functional equation (see [13], [12], and [5]) and is used for a characterization of some semigroups of operators (see [1], [7], and [17]). General solutions of

1991 Mathematics Subject Classification. Primary 39B12, 39B52; Secondary 26A18, $58 \mathrm{~F} 25$.

Key words and phrases. Cocycle, translation equation.

This paper was supported by the Silesian University Grant No. BW/IM/11/99.

ISSN 1425-6908 CHeldermann Verlag. 
(G) are well known [11] and there are some results concerning measurable and continuous solutions (see [8], also [1] and [5]).

In this paper given a compact metric space $X$ and an arbitrary function $F:(0, \infty) \times X \rightarrow X$ (not necessarily solution of $(\mathrm{T})$ ) we are concerned with solutions of $(\mathrm{G})$ defined on $(0, \infty) \times X$ which are continuous with respect to the second variable. The main result of the present paper (see Theorem 1 below) states that such a solution of $(G)$ non-vanishing and measurable with respect to the first variable is continuous. The effect "measurability implies continuity" is well known in iteration theory and functional equations in several variables. Regarding the first field, the reader is referred especially to the results concerning solutions of the translation equation (T) defined on the product of $(0, \infty)$ and an arbitrary compact metric space by M. C. Zdun for one-valued functions ([19; Theorem 1], cf. also [18; Theorem 1.1]), and by A. Smajdor for multifunctions with compact values [14; Theorem 1.2]. They proved that such a solution of $(\mathrm{T})$ measurable with respect to the first and continuous with respect to the second variable must be continuous. Moreover, one can also compare similar results for Borel measurable semigroups of continuous functions mapping paracompact finite dimensional manifold into itself [3; Theorem 4] by P. R. Chernoff and J. E. Marsden and results in operators theory, for instance in [6; Sec. 2] by E. Hille and R. S. Phillips, and [2; Proposition 2.4.2] by P. R. Chernoff. A lot of functional equations for which measurable solutions are continuous (or even smooth) can be learned from pretty general papers due to A. Járai (see, e.g. [9]). Some results of a similar type are contained in the paper [4] by K.-G. Grosse-Erdmann, where the continuity of the solution is deduced from pretty weak assumptions imposed on given functions in the equation as well as on the solution.

Proving Theorem 1 we took pattern of the argument originally used by M. C. Zdun in his paper [18] (cf. also [19]). To present it we will need some notions and facts concerning measurability and integrability of vector-valued functions.

In the whole paper $X$ denotes an arbitrary compact metric space and $\mathbb{K}$ is the set of all real or complex numbers.

Let $D$ be a set of reals and $Y$ be a metric space. A mapping $\xi: D \rightarrow Y$ is called (Lebesgue) measurable if $\xi^{-1}(U)$ is Lebesgue measurable for every open set $U \subset Y$. A measurable mapping of $D$ into $Y$ is called simple if it has only finitely many values. If $Y$ is separable then by [16; Theorem 1.9] a mapping $\xi: D \rightarrow Y$ is measurable if and only if it is the limit of a sequence of simple functions. The latter is equivalent to the notion of strong measurability considered in the monograph [6], in particular for a separable Banach space $Y$. Moreover, observe that if $Y$ is a normed space and $\xi: D \rightarrow Y$ is measurable then the function $D \ni t \mapsto\|\xi(t)\|$ is Lebesgue 
measurable as a composition of a measurable and a continuous functions. Consequently, we may also argue that a mapping $\xi$ of $D$ into a separable Banach space $Y$ is, by the definition, integrable in the Bochner sense if it is (cf. [6; Theorem 3.7.4]) measurable and the function $D \ni t \mapsto\|\xi(t)\|$ is Lebesgue integrable.

Denote by $\mathcal{C}(X, \mathbb{K})$ the set of all continuous functions mapping $X$ into $\mathbb{K}$ endowed with uniform convergence metric. It follows from the compactness of $X$ that $\mathcal{C}(X, \mathbb{K})$ is a separable Banach space (cf. [10; $\S 22 . I I I])$.

We start with the following auxiliary fact.

Lemma. Let $D$ be a set of reals at let $\xi: D \rightarrow \mathcal{C}(X, \mathbb{K})$ be such that the function $D \ni t \mapsto \xi(t)(x)$ is Lebesgue measurable for every $x \in X$. Then the mapping $\xi$ is measurable.

Proof. According to the separability of the space $\mathcal{C}(X, \mathbb{K})$ it is sufficient to show that the counterimage of an arbitrary open ball in $\mathcal{C}(X, \mathbb{K})$ is Lebesgue measurable. Fix a function $f_{0} \in \mathcal{C}(X, \mathbb{K})$ and a positive number $r$. Let $E$ be a countable dense subset of $X$. Then, due to the density of $E$,

$$
\begin{aligned}
& \xi^{-1}\left(\left\{f \in \mathcal{C}(X, \mathbb{K}):\left\|f-f_{0}\right\|<r\right\}\right) \\
& \quad=\left\{t \in D:\left\|\xi(t)-f_{0}\right\|<r\right\} \\
& \quad=\left\{t \in D: \sup \left\{\left|\xi(t)(x)-f_{0}(x)\right|: x \in X\right\}<r\right\} \\
& \quad=\left\{t \in D: \sup \left\{\left|\xi(t)(x)-f_{0}(x)\right|: x \in E\right\}<r\right\},
\end{aligned}
$$

whence, by the countability of $E$ and the assumption, it is a Lebesgue measurable set.

Let us accept the following definition. Given a function $F:(0, \infty) \times X \rightarrow$ $X$, a solution $G:(0, \infty) \times X \rightarrow \mathbb{K}$ of equation $(\mathrm{G})$ is called a cocycle of $F$ (or simply a cocycle) if for every $t \in(0, \infty)$ the function $G(t, \cdot)$ is continuous. A cocycle $G$ is said to be measurable if for every $x \in X$ the function $G(\cdot, x)$ is Lebesgue measurable.

Theorem 1. Let $G:(0, \infty) \times X \rightarrow \mathbb{K}$ be a measurable non-vanishing cocycle. Then the mapping $(0, \infty) \ni t \mapsto G(t, \cdot) \in \mathcal{C}(X, \mathbb{K})$ is continuous. In particular, the function $G$ is continuous.

Proof. Let $F:(0, \infty) \times X \rightarrow X$ be a function for which $G$ is a cocycle. Putting $f^{t}:=F(t, \cdot)$ and $g_{t}:=G(t, \cdot)$ for $t \in(0, \infty)$ we can rewrite equation $(\mathrm{G})$ in the form

$$
g_{s+t}=g_{s} \cdot g_{t} \circ f^{s} .
$$

In particular, for every $s, t \in(0, \infty)$ we have $g_{t} \circ f^{s}=g_{s+t} / g_{s} \in \mathcal{C}(X, \mathbb{K})$.

It follows from the Lemma that the mapping $(0, \infty) \ni t \mapsto g_{t} \in \mathcal{C}(X, \mathbb{K})$ is measurable. In particular, the function $(0, \infty) \ni t \mapsto\left\|g_{t}\right\|$ is Lebesgue measurable. 
Let $t \in(0, \infty)$. Observe that for every $x \in X$ we have by $(\mathrm{G})$

$$
g_{t-s} \circ f^{s}(x)=g_{t}(x) / g_{s}(x) \text { for } s \in(0, t) .
$$

Thus, by the Lemma, the mapping $(0, t) \ni s \mapsto g_{t-s} \circ f^{s}$ is measurable. In particular, the function $(0, t) \ni s \mapsto\left\|g_{t-s} \circ f^{s}\right\|$ is Lebesgue measurable.

Fix $t_{0} \in(0, \infty)$ and $\alpha, \beta \in \mathbb{R}$ such that $0<\alpha<\beta<t_{0}$. Choose an arbitrary real number $\varepsilon$ such that $|\varepsilon|<\left(t_{0}-\beta\right) / 2$. Put $a:=\left(t_{0}-\beta\right) / 2$ and $b:=t_{0}-\alpha+\left(t_{0}-\beta\right) / 2$ and observe that if $t \in\left[t_{0}-\beta, t_{0}-\alpha\right]$ then $t, t+\varepsilon \in[a, b]$.

For every $s, t \in(0, \infty)$, we have by $(\mathrm{G})$

$$
\begin{aligned}
\log \left\|g_{s+t}\right\| & =\log \left\|g_{s} \cdot g_{t} \circ f^{s}\right\| \leq \log \left(\left\|g_{s}\right\| \cdot\left\|g_{t} \circ f^{s}\right\|\right) \\
& \leq \log \left(\left\|g_{s}\right\| \cdot\left\|g_{t}\right\|\right)=\log \left\|g_{s}\right\|+\log \left\|g_{t}\right\| .
\end{aligned}
$$

Thus the function $(0, \infty) \ni t \mapsto \log \left\|g_{t}\right\|$ is subadditive. Therefore, since it is also Lebesgue measurable, in view of [6; Theorem 7.4.1] it is bounded on every compact subinterval of $(0, \infty)$, so there exists an $M>0$ such that

$$
\left\|g_{t}\right\| \leq M \quad \text { for } t \in[\alpha, \beta] \cup[a, b] .
$$

Then, by (G) and (1), we have

$$
\begin{aligned}
\left\|g_{t_{0}+\varepsilon}-g_{t_{0}}\right\| & =\frac{1}{\beta-\alpha} \int_{\alpha}^{\beta}\left\|g_{t_{0}+\varepsilon}-g_{t_{0}}\right\| d s \\
& =\frac{1}{\beta-\alpha} \int_{\alpha}^{\beta}\left\|g_{s} \cdot g_{t_{0}+\varepsilon-s} \circ f^{s}-g_{s} \cdot g_{t_{0}-s} \circ f^{s}\right\| d s \\
& =\frac{1}{\beta-\alpha} \int_{\alpha}^{\beta}\left\|g_{s} \cdot\left(g_{t_{0}+\varepsilon-s}-g_{t_{0}-s}\right) \circ f^{s}\right\| d s \\
& \leq \frac{1}{\beta-\alpha} \int_{\alpha}^{\beta}\left\|g_{s}\right\| \cdot\left\|\left(g_{t_{0}+\varepsilon-s}-g_{t_{0}-s}\right) \circ f^{s}\right\| d s \\
& \leq \frac{M}{\beta-\alpha} \int_{\alpha}^{\beta}\left\|\left(g_{t_{0}+\varepsilon-s}-g_{t_{0}-s}\right) \circ f^{s}\right\| d s \\
& \leq \frac{M}{\beta-\alpha} \int_{\alpha}^{\beta}\left\|g_{t_{0}+\varepsilon-s}-g_{t_{0}-s}\right\| d s \\
& =\frac{M}{\beta-\alpha} \int_{t_{0}-\beta}^{t_{0}-\alpha}\left\|g_{t+\varepsilon}-g_{t}\right\| d t,
\end{aligned}
$$

that is

$$
\left\|g_{t_{0}+\varepsilon}-g_{t_{0}}\right\| \leq \frac{M}{\beta-\alpha} \int_{t_{0}-\beta}^{t_{0}-\alpha}\left\|g_{t+\varepsilon}-g_{t}\right\| d t .
$$

Now define

$$
\bar{g}_{t}:= \begin{cases}g_{t}, & t \in[a, b], \\ 0, & t \in \mathbb{R} \backslash[a, b] .\end{cases}
$$


Since the mapping $(0, \infty) \ni t \mapsto g_{t}$ is measurable so is the mapping $(0, \infty) \ni$ $t \mapsto \bar{g}_{t}$. Moreover,

$$
\int_{t_{0}-\beta}^{t_{0}-\alpha}\left\|g_{t+\varepsilon}-g_{t}\right\| d t=\int_{t_{0}-\beta}^{t_{0}-\alpha}\left\|\bar{g}_{t+\varepsilon}-\bar{g}_{t}\right\| d t \leq \int_{\mathbb{R}}\left\|\bar{g}_{t+\varepsilon}-\bar{g}_{t}\right\| d t
$$

that is, by (2),

$$
\left\|g_{t_{0}+\varepsilon}-g_{t_{0}}\right\| \leq \frac{M}{\beta-\alpha} \int_{\mathbb{R}}\left\|\bar{g}_{t+\varepsilon}-\bar{g}_{t}\right\| d t
$$

for each $\varepsilon$ satisfying $|\varepsilon|<\left(t_{0}-\beta\right) / 2$. Further (cf. (1)) $\left\|\bar{g}_{t}\right\|=\left\|g_{t}\right\| \leq M$ for $t \in[a, b]$ and $\left\|\bar{g}_{t}\right\|=0$ otherwise and the integral $\int_{\mathbb{R}}\left\|\bar{g}_{t}\right\| d t$ is finite, whence we infer that the function $\mathbb{R} \ni t \mapsto \bar{g}_{t}$ is integrable in the Bochner sense. Using [6; Theorem 3.8.3] we obtain

$$
\lim _{\varepsilon \rightarrow 0} \int_{\mathbb{R}}\left\|\bar{g}_{t+\varepsilon}-\bar{g}_{t}\right\| d t=0
$$

which due to (3) shows that

$$
\lim _{\varepsilon \rightarrow 0}\left\|g_{t_{0}+\varepsilon}-g_{t_{0}}\right\|=0 .
$$

This means that the mapping $(0, \infty) \ni t \mapsto G(t, \cdot) \in \mathcal{C}(X, \mathbb{K})$ is continuous at $t_{0}$.

Observe that the continuity of the mapping $t \mapsto G(t, \cdot)$ means that the function $G$ is continuous with respect to the first variable, uniformly with respect to the second one. Hence and by continuity of $G$ with respect to the second variable, we may infer that $G$ is continuous.

Proposition 1. Let $\Omega$ be a set, $F:(0, \infty) \times \Omega \rightarrow \Omega$ be a function and let $G:(0, \infty) \times \Omega \rightarrow \mathbb{K}$ be a solution of equation $(G)$. If $\lim _{t \rightarrow 0} G(t, x)=1$ for every $x \in \Omega$ then the function $G(\cdot, x)$ is right continuous for every $x \in \Omega$.

Proof. Fix $x \in \Omega$ and $s \in(0, \infty)$. Since $\lim _{t \rightarrow 0} G(t, F(s, x))=1$, it follows from $(\mathrm{G})$ that

$$
\begin{aligned}
|G(s+t, x)-G(s, x)| & =|G(s, x) G(t, F(s, x))-G(s, x)| \\
& =|G(s, x)||G(t, F(s, x))-1|
\end{aligned}
$$

for every $t \in(0, \infty)$ which converges to zero whenever $t \rightarrow 0$.

Theorem 1 and Proposition 1 imply the following.

Corollary 1. Let $G:(0, \infty) \times X \rightarrow \mathbb{K}$ be a non-vanishing cocycle. Assume that $\lim _{t \rightarrow 0} G(t, x)=1$ for every $x \in X$. Then the mapping $(0, \infty) \ni t \mapsto$ $G(t, \cdot) \in \mathcal{C}(X, \mathbb{K})$ is continuous. In particular, the function $G$ is continuous. 
Proof. By Proposition 1 for every $x \in X$ the function $G(\cdot, x)$ is right continuous so Lebesgue measurable and the assertion follows from Theorem 1.

Now we will consider positive cocycles.

Theorem 2. Let $G:(0, \infty) \times X \rightarrow(0, \infty)$ be a cocycle. Assume that either $G<1$, or $G>1$. Then for each $x \in X$ the function $G(\cdot, x)$ is strictly decreasing in the case $G<1$, strictly increasing in the case $G>1$ and the mapping $(0, \infty) \ni t \mapsto G(t, \cdot) \in \mathcal{C}(X, \mathbb{R})$ is continuous. In particular, the function $G$ is continuous.

Proof. Let $F:(0, \infty) \times X \rightarrow X$ be a function for which $G$ is a cocycle and fix an $x \in X$. We will show that the function $G(\cdot, x)$ is strictly monotonic. Let $s, t \in(0, \infty)$ and $s<t$. Then, using $(\mathrm{G})$, we have

$$
G(t, x)=G(s, x) G(t-s, F(s, x)) .
$$

Thus, if $G<1$ then $G(t, x)<G(s, x)$ and, consequently, $G(\cdot, x)$ is strictly decreasing, and if $G>1$ then $G(t, x)>G(s, x)$ and $G(\cdot, x)$ is strictly increasing. Therefore $G$ is a measurable cocycle and it is enough to apply Theorem 1 to complete the proof.

Proposition 2. Let $\Omega$ be a metric space, $F:(0, \infty) \times \Omega \rightarrow \Omega$ be a function and let $G:(0, \infty) \times \Omega \rightarrow \mathbb{K}$ be a solution of equation $(G)$, and $\left(t_{0}, x_{0}\right) \in$ $(0, \infty) \times \Omega$. Assume that $\lim _{t \rightarrow 0} F\left(t, x_{0}\right)=x_{0}$, the function $G\left(\cdot, x_{0}\right)$ is right continuous at $t_{0}$ and the function $G\left(t_{0}, \cdot\right)$ is continuous at $x_{0}$. If there exists $\lim _{t \rightarrow 0} G\left(t, x_{0}\right)$ then either

$$
G\left(t_{0}, x_{0}\right)=0
$$

or

$$
\lim _{t \rightarrow 0} G\left(t, x_{0}\right)=1
$$

Proof. Assume that there exists $\lim _{t \rightarrow 0} G\left(t, x_{0}\right)=: e\left(x_{0}\right)$ and $G\left(t_{0}, x_{0}\right) \neq 0$. According to the right continuity of $G\left(\cdot, x_{0}\right)$ at $t_{0}$, continuity of $G\left(t_{0}, \cdot\right)$ at $x_{0}$ and equation $(\mathrm{G})$ we have

$G\left(t_{0}, x_{0}\right)=\lim _{t \rightarrow 0+} G\left(t_{0}+t, x_{0}\right)=\lim _{t \rightarrow 0} G\left(t, x_{0}\right) G\left(t_{0}, F\left(t, x_{0}\right)\right)=e\left(x_{0}\right) G\left(t_{0}, x_{0}\right)$.

Therefore, since $G\left(t_{0}, x_{0}\right) \neq 0$ we have $e\left(x_{0}\right)=1$.

Using Theorem 2 and Proposition 2 we come to the following. 
Corollary 2. Let $F:(0, \infty) \times X \rightarrow X$ be a function and $G:(0, \infty) \times X \rightarrow$ $(0, \infty)$ be its cocycle such that either $G<1$, or $G>1$. Then for every $x \in X$ there exists $\lim _{t \rightarrow 0} G(t, x)$. If, in addition, $x_{0} \in X$ and $\lim _{t \rightarrow 0} F\left(t, x_{0}\right)=$ $x_{0}$ then $\lim _{t \rightarrow 0} G\left(t, x_{0}\right)=1$ and $G\left(\cdot, x_{0}\right)$ is a decreasing homeomorphism mapping $(0, \infty)$ onto $(0,1)$ in the case $G<1$ and $G\left(\cdot, x_{0}\right)$ is an increasing homeomorphism mapping $(0, \infty)$ onto $(1, \infty)$ in the case $G>1$.

Proof. Assume that $G<1$. Then, by Theorem $2, G(\cdot, x)$ is strictly decreasing and continuous for every $x \in X$. In particular, for every $x \in X$ there exists $\lim _{t \rightarrow 0} G(t, x)$.

Fix an $x_{0} \in X$ and assume that $\lim _{t \rightarrow 0} F\left(t, x_{0}\right)=x_{0}$. Then, by virtue of Proposition 2, $\lim _{t \rightarrow 0} G\left(t, x_{0}\right)=1$. To complete the proof it is enough to prove that $\lim _{t \rightarrow \infty} G\left(t, x_{0}\right)=0$. To this aim, taking into account the compactness of $X$, choose a strictly increasing sequence $\left(s_{n}: n \in \mathbb{N}\right)$ of positive integers such that the sequence $\left(F\left(s_{n}, x_{0}\right): n \in \mathbb{N}\right)$ converges to an $\bar{x} \in X$. In particular, we have $\lim _{n \rightarrow \infty} s_{n}=\infty$. Using equation (G) we have

$$
G\left(s_{n}+1, x_{0}\right)=G\left(s_{n}, x_{0}\right) G\left(1, F\left(s_{n}, x_{0}\right)\right),
$$

whence, due to the continuity of $G(1, \cdot)$ at $\bar{x}$,

$$
\lim _{t \rightarrow \infty} G\left(t, x_{0}\right)=\lim _{t \rightarrow \infty} G\left(t, x_{0}\right) G(1, \bar{x}) .
$$

Consequently, since $0<G(1, \bar{x})<1$, we obtain $\lim _{t \rightarrow \infty} G\left(t, x_{0}\right)=0$.

Now assume that $G>1$. Then $1 / G<1$. Moreover, $1 / G$ is also a cocycle of $F$ so by the first part of the proof there exists $\lim _{t \rightarrow 0} 1 / G(t, x)$. Hence there exists $\lim _{t \rightarrow 0} G(t, x)$. In the same way we may infer that the last statement of Corollary 2 holds.

\section{References}

[1] Arendt, W., Characterizations of positive semigroups on $C_{0}(X)$, in: "One Parameter Semigroups of Positive Operators", Lecture Notes in Math. 1184, Springer-Verlag, Berlin-New York, 1986, 122-162.

[2] Chernoff, P. R., Product formulas, nonlinear semigroups and addition of unbounded operators, Mem. Amer. Math. Soc. 140 (1974).

[3] Chernoff, P. R., Marsden, J. E., On continuity and smoothness of group action, Bull. Amer. Math. Soc. 76 (1970), 1044-1049.

[4] Grosse-Erdmann, K.-G., Regularity properties of functional equations and inequalities, Aequationes Math. 37 (1989), 233-251.

[5] Guzik, G., On embedding of linear functional equation, Rocznik Nauk. Dyd. WSP w Krakowie 207 (1999), 23-33.

[6] Hille, E., Phillips, R. S., Functional Analysis and Semigroups, Amer. Math. Soc. Colloq. Publ., Providence, Rhode Island, 1957. 
[7] Hmissi, M., Sur l'équation fonctionnelle des cocycles d'un système semidynamique, in: European Conference on Iteration Theory, Batschuns 1989, World Scientific, Singapore, 1991, 149-156.

[8] Hmissi, M., Sur les solutions globales de l'équation des cocycles, Aequationes Math. 45 (1993), 195-206.

[9] Járai, A., On regular solutions of functional equations, Aequationes Math. 30 (1986), $21-54$.

[10] Kuratowski, K., Topology Vol. I, Academic Press and Polish Scientific Publishers, New York and Warszawa, 1966.

[11] Moszner, Z., Structure de l'automate plein, réduit et inversible, Aequationes Math. 9 (1973), 46-59.

[12] Moszner, Z., Sur le prolongement covariant d'une équation linéaire par rapport au groupe d'itération, Sitzungsber, Abt. II 207 (1998), 173-182.

[13] Reich, L., 24 Remark, in: The Thirty-Fifth International Symposium on Functional Equations, September 7-14, 1997, Graz-Mariatrost, Austria, Aequationes Math. 55 (1998), 311-312.

[14] Smajdor, A., Iterations of multi-valued functions, Prace Nauk. Uniw. Śląskiego 759 (1983), Katowice.

[15] Totoki, H., Time changes of flows, Mem. Fac. Sci. Kyushu Univ. Ser. A 20 (1966), 26-55.

[16] Vakhaniya, N.N., Tarieladze, V.I., Chobanyan, S.A., Probability Distributions in Banach Spaces (in Russian), Nauka, Moscow, 1985.

[17] Vesentini, E., Semiflows and semigroups, Rend. Mat. Accad. Lincei (9) 7 (1996), 75-82.

[18] Zdun, M. C., Continuous and differentiable iteration semigroups, Prace Nauk. Uniw. Śląskiego 308 (1979), Katowice.

[19] Zdun, M. C., On continuity of iteration semigroups on metric space, Comment. Math. 29 (1989), 113-116.

GrZEGorz GuZIK

INSTITUTE OF MATHEMATICS

SILESIAN UNIVERSITY

BANKOWA 14

40-007 Katowice, Poland 